\title{
BMJ Open Do quality improvement initiatives for diabetes care address social inequities? Secondary analysis of a systematic review
}

\author{
Jacquie Boyang Lu, ${ }^{1}$ Kristin J Danko, ${ }^{2,3}$ Michael D Elfassy, ${ }^{4}$ Vivian Welch, ${ }^{3,5}$ \\ Jeremy M Grimshaw, ${ }^{2,3,6}$ Noah M Ivers ${ }^{7,8,9}$
}

To cite: Lu JB, Danko KJ, Elfassy MD, et al. Do quality improvement initiatives for diabetes care address social inequities? Secondary analysis of a systematic review. BMJ Open 2018;8:e018826. doi:10.1136/ bmjopen-2017-018826

- Prepublication history and additional material for this paper are available online. To view these files, please visit the journal online (http://dx.doi. org/10.1136/bmjopen-2017018826).

Received 29 July 2017 Revised 20 December 2017 Accepted 2 January 2018

Check for updates

For numbered affiliations see end of article.

Correspondence to Jacquie Boyang Lu; jacquie.lu@queensu.ca

\section{ABSTRACT}

Background Socially disadvantaged populations carry a disproportionate burden of diabetes-related morbidity and mortality. There is an emerging interest in quality improvement (Ql) strategies in the care of patients with diabetes, however, the effect of these interventions on disadvantaged groups remains unclear.

Objective This is a secondary analysis of a systematic review that seeks to examine the extent of equity considerations in diabetes QI studies, specifically quantifying the proportion of studies that target interventions toward disadvantaged populations and conduct analyses on the impact of interventions on disadvantaged groups.

Research design and methods Studies were identified using Medline, HealthStar and the Cochrane Effective Practice and Organisation of Care database. Randomised controlled trials assessing $12 \mathrm{Ql}$ strategies targeting health systems, healthcare professionals and/or patients for the management of adult outpatients with diabetes were eligible. The place of residence, race/ethnicity/culture/ language, occupational status, gender/sexual identity, religious affiliations, education level, socioeconomic status, social capital, plus age, disability, sexual preferences and relationships (PROGRESS-Plus) framework was used to identify trials that focused on disadvantaged patient populations, to examine the types of equity-relevant factors that are being considered and to explore temporal trends in equity-relevant diabetes QI trials.

Results of the 278 trials that met the inclusion criteria, 95 trials had equity-relevant considerations. These include 64 targeted trials that focused on a disadvantaged population with the aim to improve the health status of that population and 31 general trials that undertook subgroup analyses to assess the extent to which their interventions may have had differential impacts on disadvantaged subgroups. Trials predominantly focused on race/ethnicity, socioeconomic status and place of residence as potential factors for disadvantage in patients receiving diabetes care.

Conclusions Less than one-third of diabetes QI trials included equity-relevant considerations, limiting the relevance and applicability of their data to disadvantaged populations. There is a need for better data collection, reporting, analysis and interventions on the social determinants of health that may influence the health outcomes of patients with diabetes.
Strengths and limitations of this study

- The use of the 'place of residence, race/ethnicity/ culture/language, occupational status, gender/ sexual identity, religious affiliations, education level, socioeconomic status, social capital, plus age, disability, sexual preferences and relationships' framework provides a standardised approach for identifying and analysing equity-relevant information within research publications. The focus on only primary publications of trials meant any equity-relevant analyses in subsequent publications of the same trial were not captured in this review.

- The lack of standardised terminology for equityrelevant information in the general literature restricted our ability to fully capture all the various issues that may lead to disadvantages in medical care.

- The inclusion of only English language publications was a practical limitation on the scope of this study.

PROSPERO registration number CRD42013005165.

\section{INTRODUCTION}

Diabetes affects approximately $8.5 \%$ of the adult population; the increasing prevalence of physical inactivity, obesity and an ageing population means that this number will increase over time. ${ }^{12}$ The burden of diabetes is not evenly spread through the population. Racial and ethnic minorities, as well as people of lower socioeconomic status are more likely to develop diabetes. ${ }^{23}$ Such individuals are also more likely to experience delayed diagnosis and lower quality of care, leading to greater risk for diabetes-related complications. $^{23}$

Patients with diabetes require lifelong medications, routine follow-up with healthcare professionals and regular preventative screening exams to reduce the likelihood of morbidity and mortality. ${ }^{4} 5$ 
Socially disadvantaged groups may experience multiple barriers to high quality care due to factors such as differences in language or culture, inadequate financial resources or prohibitive distances from care centres. ${ }^{26-8}$ To ensure that innovations and standards of care in healthcare reach the most disadvantaged segments of the population, interventions must recognise and address these equity-based considerations. $^{27-9}$

Quality improvement (QI) in the care of patients with diabetes is a rapidly expanding field of interest. ${ }^{10}$ However, while many QI strategies are effective in improving diabetes care in general populations, ${ }^{10}$ it is unclear whether they improve or worsen health disparities among disadvantaged subgroups. ${ }^{11}$ QI strategies designed for the general population may not be accessible to disadvantaged groups or may not have the same efficacy in disadvantaged populations and may inadvertently lead to an increase in diabetes-related health disparities. ${ }^{2791112}$

Recently, we updated a systematic review of trials of QI strategies for diabetes care. ${ }^{13}$ In this secondary analysis of that review, we examine the extent to which health equity concerns were considered in diabetes QI studies. Specifically, we quantified the proportion of QI studies that targeted interventions toward disadvantaged populations, looked at risk factors for disadvantage in the patient population and analysed the impact of interventions on disadvantaged subgroups.

\section{RESEARCH DESIGN AND METHODS}

Our goal was to examine the extent of focus in the literature on understanding the effects of diabetes QI for disadvantaged populations. A detailed description of methods used for searching and screening the relevant data for the underlying systematic review has been published. ${ }^{13}$

\section{Study selection and data extraction}

An experienced librarian developed the search strategy, which was peer reviewed independently by another information specialist. Studies were identified using Medline, HealthStar and the Cochrane Effective Practice and Organisation of Care database. Randomised controlled trials (RCTs) assessing 1 of the 12 predefined QI strategies targeting health systems, healthcare professionals and/or patients for the management of adult outpatients with diabetes were eligible. Studies had to report at least one process of care measure (eg, proportion of patients taking acetylsalicylic acid, statins or antihypertensive medication; screened for retinopathy or foot abnormalities and monitored for renal function) or intermediate outcomes (eg, glycosylated haemoglobin levels (HbAlc), low-density lipoprotein cholesterol levels, diastolic and systolic blood pressure; proportion of patients with controlled hypertension or who quit smoking).

For this secondary review, we developed a data extraction form using PROGRESS-Plus framework, ${ }^{9}$ to consider the range of factors that may increase the risk for a population subgroup to be disadvantaged, including participants' place of residence, race/ethnicity/culture/ language, occupational status, gender/sexual identity, religious affiliations, education level, socioeconomic status, social capital, plus age, disability, sexual preferences and relationships. ${ }^{9}$ Specifically, we interpreted these risk factors in the context of a patient's ability to access healthcare and effectively manage their diabetes. For example, it may be more difficult for patients living in rural or geographically isolated areas to access dependable primary care, leading to negative implications for their ability to achieve diabetes-related targets. Online supplementary table 1 outlines our full interpretation of PROGRESS-Plus factors in considering how these factors might lead to inequity in diabetes management across subpopulations, developed based on previous literature, ${ }^{6}$ PROGRESS framework, ${ }^{9}$ and in collaboration with PROGRESS authors (VW).

Two reviewers independently extracted data based on a thorough reading of the full text for all included studies. This allowed us to identify a cohort of equity-relevant trials that targeted or assessed equity factors as defined by PROGRESS-Plus framework. ${ }^{9}$ Within this cohort, trials were classified as either Targeted or General. Targeted equity-relevant trials were defined as a trial focused on a population with an identified disadvantage in health in order to improve the health status of that population or to reduce the health gradient. General equity-relevant trials were defined as a trial involved a broad participant population but made comparisons of effects in disadvantaged subgroups to assess the extent to which interventions may have differential impacts. For example, a trial testing a primary care-based culturally sensitive behavioural intervention in a population of urban African-Americans with type 2 diabetes ${ }^{14}$ would be classified as a targeted equity-relevant trial because it purposefully directed interventions towards a disadvantaged population. In contrast, a trial testing a tele-homecare monitoring system for patients with type 2 diabetes that explored the benefit of the intervention in female patients and those with lower education levels, ${ }^{15}$ would be classified as a general equity-relevant trial.

The reviewers extracted PROGRESS-Plus factors identified in the baseline patient characteristics for all studies. Additionally, for equity-relevant studies, the reviewers extracted PROGRESS-Plus factors identified in the study objective, study design (eg, patient eligibility criteria and patient recruitment techniques) and analysis of results. We only coded instances when authors were explicit in their mention of PROGRESS-Plus factors. Discrepancies were resolved by discussion or the involvement of authors NMI and KJD.

\section{Data synthesis and analysis}

As we had no a priori hypothesis about the differences or similarities that would be found among trials regarding issues of equity or efforts to address areas of disadvantage, we provide here descriptive analyses only. We describe the proportion of trials that focused on equity-relevant 


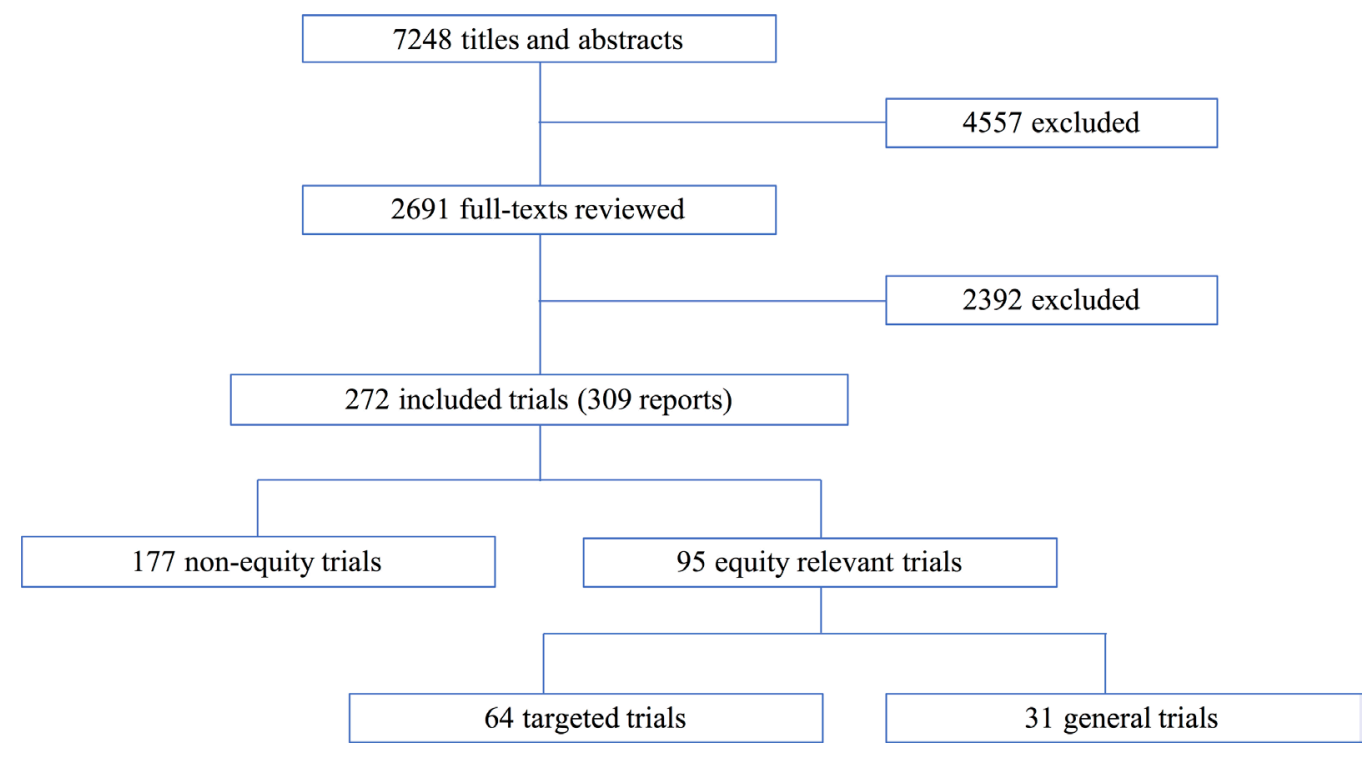

Figure 1 Preferred Reporting Items for Systematic Reviews and Meta-Analyses flow diagram.

factors and types of factors considered in these trials. In addition, to explore for time-trends in the consideration of equity-relevant factors in diabetes QI trials, we split our analyses by median date of study conduct.

\section{RESULTS}

\section{Literature search and review process}

Figure 1 summarises the flow of literature in the QI review. The initial search identified a total of 7248 citations; review of 2691 full text articles resulted in a final sample of 309 reports, representing 272 unique trials.

\section{Study characteristics}

Ninety-five trials $(34.9 \%)$ were identified as equity-relevant; 64 of these were classified as targeted and 31 as general. Characteristics were similar between non-equity studies and equity-relevant studies (table 1). Most trials focused on patients with type 2 diabetes and looked at glycaemic control combined with cardiovascular status or other health benchmarks (aspirin use, statin use, hypertensive drug use, screening for retinopathy/nephropathy/neuropathy and smoking cessation) as the primary outcomes of interest. Mean baseline HbAlc was comparable between non-equity and equity-relevant trials, but targeted trials reported the inclusion of a greater proportion of patients with $\mathrm{HbA1c} \geq 8 \%$ compared with general trials.

Interestingly, $73.7 \%$ of equity-relevant trials were based in countries with private health insurance systems, whereas $32.6 \%$ of equity-relevant trials were based in countries with universal health insurance. However, this finding may simply reflect the predominance of publications from the USA included within this review, contributing 65 of 177 non-equity studies and 63 of 95 equity-relevant studies.

The relative frequency of individual QI strategies assessed in the studies were also comparable across non-equity and equity-relevant trials with a few exceptions. Overall, patient education, promotion of self-management and case management were the most frequently used QI interventions across all studies. Among equity-relevant trials, case management, team changes and patient education were evaluated more frequently in targeted trials compared with general trials.

Table 2 describes the frequency of PROGRESS-Plus factors examined in all studies. Among 177 non-equity studies, $94.4 \%$ reported data on the age of participants, $90.4 \%$ reported data on gender/sex, $35.0 \%$ reported data on race/ethnicity/culture/language, $32.2 \%$ reported data on education levels and $29.9 \%$ reported data on disability status. Overall, age and gender/sex were the most frequently documented PROGRESS-Plus factors, appearing equally in both equity-relevant studies and non-equity studies. In comparison, race/ethnicity/ culture/language, socioeconomic status, education, social capital, occupation and place of residence appeared significantly more frequently in equity-relevant trials than in non-equity trials. PROGRESS-Plus factors of sexual preference, features of relationships and time-dependent relationships were not identified in any of the studies.

The targeted trials were most commonly directed toward race/ethnicity/language/culture $\quad(53.1 \%$ of targeted trials), place of residence $(29.7 \%)$ and socioeconomic status $(28.1 \%)$; occupation, gender/sex, religion or social capital were rarely addressed in the objectives of target trials. Most targeted trials used a single PROGRESS-Plus factor to define its patient population (eg, interventions targeted people in rural communities or patients belonging to a particular ethnic minority). Twenty trials looked at population subgroups with two PROGRESS-Plus factors. Only two trials simultaneously targeted three factors. ${ }^{1617}$ Fifteen targeted trials $(23 \%)$ conducted sub-analyses to understand whether their intervention varied in its effectiveness across additional 
Table 1 Study characteristics

\begin{tabular}{|c|c|c|c|c|}
\hline \multirow[b]{2}{*}{ Study characteristics } & \multirow{2}{*}{$\begin{array}{l}\text { Non-equity studies, } \\
\mathrm{n}=177\end{array}$} & \multicolumn{3}{|c|}{ Equity-relevant studies } \\
\hline & & All, $n=95$ & Targeted, $\mathrm{n}=64$ & General, n=31 \\
\hline Sample size & $931.6(17-23740)$ & $733.5(35-7557)$ & $490.7(35-7557)$ & $1226.8(46-7009)$ \\
\hline Duration of follow-up (months) & $14(3-159.6)$ & $13.2(3-72)$ & $12.5(3-60)$ & $14.6(3-72)$ \\
\hline \multicolumn{5}{|l|}{ Types of diabetes } \\
\hline Type 1 & $9(5.1)$ & $5(5.3)$ & $1(1.6)$ & $4(12.9)$ \\
\hline Type 2 & $116(65.5)$ & $51(53.7)$ & $37(57.8)$ & $14(45.2)$ \\
\hline Types 1 and 2 & $0(0)$ & $17(17.9)$ & $9(14.1)$ & $8(25.8)$ \\
\hline Type unclear or not reported & $19(10.7)$ & $22(23.2)$ & $17(26.6)$ & $5(16.1)$ \\
\hline \multicolumn{5}{|l|}{ Mean baseline HbA1c } \\
\hline$<8 \%$ or $64 \mathrm{mmol} / \mathrm{mol}$ & $56(31.6)$ & $33(34.7)$ & $19(29.7)$ & $14(45.2)$ \\
\hline$\geq 8 \%$ or $63 \mathrm{mmol} / \mathrm{mol}$ & $90(50.8)$ & $45(47.4)$ & $34(53.1)$ & $11(35.5)$ \\
\hline Not reported & $31(17.5)$ & $17(17.9)$ & $11(17.2)$ & $6(19.4)$ \\
\hline \multicolumn{5}{|l|}{ Primary focus } \\
\hline Glycaemic only & $44(24.9)$ & $19(20)$ & $8(12.5)$ & $11(35.5)$ \\
\hline Glycaemic and CVD & $45(25.4)$ & 30 (31.6) & $21(32.8)$ & $9(29)$ \\
\hline Glycaemic and other & $0(0)$ & $33(34.7)$ & $25(39.1)$ & $8(25.8)$ \\
\hline CVD only & $16(9)$ & $6(6.3)$ & $4(6.3)$ & $2(6.5)$ \\
\hline Other or unclear & $8(4.5)$ & $7(7.4)$ & $6(9.4)$ & $1(3.2)$ \\
\hline \multicolumn{5}{|l|}{ Country of study by health system } \\
\hline Universal healthcare & $96(54.2)$ & $31(32.6)$ & $12(18.8)$ & 19 (61.3) \\
\hline Private health insurance & $79(44.6)$ & $70(73.7)$ & $52(81.2)$ & $18(58.1)$ \\
\hline \multicolumn{5}{|l|}{ Intervention methods } \\
\hline$A F$ & $29(16.4)$ & $17(9.6)$ & $10(5.6)$ & $7(4)$ \\
\hline $\mathrm{CM}$ & $110(62.1)$ & $71(40.1)$ & 53 (29.9) & 18 (10.2) \\
\hline TC & $74(41.8)$ & $49(27.7)$ & 35 (19.8) & $14(7.9)$ \\
\hline EPR & $48(27.1)$ & $24(13.6)$ & $10(5.6)$ & $14(7.9)$ \\
\hline CE & $72(40.7)$ & $31(17.5)$ & $23(13)$ & $8(4.5)$ \\
\hline CR & $35(19.8)$ & $29(16.4)$ & $10(5.6)$ & $19(10.7)$ \\
\hline FR & $73(41.2)$ & $30(16.9)$ & $12(6.8)$ & $18(10.2)$ \\
\hline PE & 165 (93.2) & $98(55.4)$ & $74(41.8)$ & $24(13.6)$ \\
\hline PSM & $153(86.4)$ & $81(45.8)$ & $54(30.5)$ & 27 (15.3) \\
\hline PR & $35(19.8)$ & $25(14.1)$ & $13(7.3)$ & $12(6.8)$ \\
\hline CQI & $9(5.1)$ & $1(0.6)$ & $1(0.6)$ & $0(0)$ \\
\hline $\mathrm{FI}$ & $6(3.4)$ & $6(3.4)$ & $4(2.3)$ & $2(1.1)$ \\
\hline
\end{tabular}

Sample size and duration of follow-up reported as mean (range). All other categories reported as $\mathrm{n}$ (\%). Under primary focus, other refers to aspirin use, statin use, hypertensive drug use, smoking cessation, as well as screening for retinopathy, nephropathy or neuropathy. Countries with universal healthcare include: Argentina, Australia, Austria, Belgium, Brazil, Canada, Denmark, Finland, France, Germany, Greece, Hong Kong, Iceland, Ireland, Israel, Italy, Japan, Luxembourg, New Zealand, Norway, Portugal, Singapore, South Korea, Spain, Switzerland, The Netherlands, UAE and UK. Countries with privatized health insurance include: China, India, Iran, Jordan, Mexico, Oman, Poland, South Africa, Thailand, Turkey and USA. Two trials were conducted over multiple countries, in which case each country was counted as a discrete entity.

$\mathrm{AF}$, audit and feedback; CE, clinician education; CM, case management; CQI, continuous quality improvement; CR, clinician reminders; CVD, cardiovascular disease; DM, diabetes mellitus; EPR, electronic patient registry; FI, financial incentives; FR, facilitated relay; HbA1c, glycated haemoglobin; PE, patient education; PR, patient reminders; PSM, promotion of self-management; TC, team changes.

PROGRESS-Plus factors distinct from those used to define their intervention and their target patient populations.

Of the 31 general trials that did not contain an equity-specific objective but conducted stratified analyses across PROGRESS-Plus factors, gender/sex $(71.0 \%$ of general trials), age $(71.0 \%)$, race/ethnicity/culture/ language $(25.8 \%)$ and education $(29.0 \%)$ were the most commonly stratified factors. Twelve trials found 
Table 2 PROGRESS-Plus factors by trial type

\begin{tabular}{|c|c|c|c|c|c|c|c|c|c|c|}
\hline \multirow{3}{*}{$\begin{array}{l}\text { PROGRESS- } \\
\text { Plus factors }\end{array}$} & \multirow{3}{*}{$\begin{array}{l}\text { Non-equity } \\
\text { studies, } \\
\mathrm{n}=177\end{array}$} & \multicolumn{3}{|c|}{ Equity-relevant studies } & \multicolumn{6}{|c|}{ Equity-relevant studies, n=95 } \\
\hline & & \multirow{2}{*}{$\begin{array}{l}\text { All, } \\
\mathrm{n}=95\end{array}$} & \multirow{2}{*}{$\begin{array}{l}\text { Targeted, } \\
\mathrm{n}=64\end{array}$} & \multirow{2}{*}{$\begin{array}{l}\text { General, } \\
\mathrm{n}=\mathbf{3 1}\end{array}$} & \multicolumn{3}{|c|}{ Targeted, $\mathrm{n}=64$} & \multicolumn{3}{|c|}{ General, $n=31$} \\
\hline & & & & & 0 & B & A & 0 & B & A \\
\hline $\begin{array}{l}\text { Place of } \\
\text { residence }\end{array}$ & $4(2.3)$ & $19(20)$ & $19(29.7)$ & $0(0)$ & $19(29.7)$ & $1(1.6)$ & $0(0)$ & $0(0)$ & $0(0)$ & $0(0)$ \\
\hline $\begin{array}{l}\text { Race/ethnicity/ } \\
\text { culture/language }\end{array}$ & $62(35)$ & $75(78.9)$ & $57(89.1)$ & $18(58.1)$ & $34(53.1)$ & $41(64.1)$ & $7(10.9)$ & $0(0)$ & $18(58.1)$ & $8(25.8)$ \\
\hline Occupation & $17(9.6)$ & $24(25.3)$ & $18(28.1)$ & $6(19.4)$ & $1(1.6)$ & $17(26.6)$ & $1(1.6)$ & $0(0)$ & $6(19.4)$ & $2(6.5)$ \\
\hline Gender/sex & $160(90.4)$ & $91(95.8)$ & $60(93.8)$ & $31(100)$ & $0(0)$ & $60(93.8)$ & $9(14.1)$ & $0(0)$ & $31(100)$ & $22(71)$ \\
\hline Religion & $0(0)$ & $1(1.1)$ & $1(1.6)$ & $0(0)$ & $1(1.6)$ & $0(0)$ & $0(0)$ & $0(0)$ & $0(0)$ & $0(0)$ \\
\hline Education & $57(32.2)$ & $57(60)$ & $42(65.6)$ & $15(48.4)$ & $1(1.6)$ & $42(65.6)$ & $5(7.8)$ & $0(0)$ & $14(45.2)$ & $9(29)$ \\
\hline $\begin{array}{l}\text { Socioeconomic } \\
\text { status }\end{array}$ & $27(15.3)$ & $51(53.7)$ & $45(70.3)$ & $6(19.4)$ & $18(28.1)$ & $40(62.5)$ & $2(3.1)$ & $0(0)$ & $5(16.1)$ & $2(6.5)$ \\
\hline Social capital & 30 (16.9) & $33(34.7)$ & $30(46.9)$ & $3(9.7)$ & $0(0)$ & $30(46.9)$ & $1(1.6)$ & $0(0)$ & $3(9.7)$ & $0(0)$ \\
\hline Plus 1. age & $167(94.4)$ & $92(96.8)$ & 61 (95.3) & $31(100)$ & $8(12.5)$ & 61 (95.3) & $7(10.9)$ & $0(0)$ & 31 (100) & $22(71)$ \\
\hline Plus 1. disability & $53(29.9)$ & $32(33.7)$ & $23(35.9)$ & $9(29)$ & 7 (10.9) & $22(34.4)$ & $3(4.7)$ & $0(0)$ & $9(29)$ & $1(3.2)$ \\
\hline
\end{tabular}

All values are expressed as $n$ (\%). O, study objective; B, baseline patient characteristics; A, study analysis. PROGRESS-Plus factors of sexual preference, features of relationships and time-dependent relationships were omitted from this table as we did not find any studies which looked at these characteristics as a potential risk factor for being disadvantaged.

Each trial is counted once under each column. Columns on the left of the table reflect the number of trials that contained PROGRESS-Plus factors in the objectives $(O)$, baseline characteristics $(B)$ or analysis (A). Trials that assessed for PROGRESS-Plus factors in two or more categories of $\mathrm{O}, \mathrm{B}$ and $\mathrm{A}$ were still counted as one trial. As such, the sum of trials under headings $\mathrm{O}$, B and $\mathrm{A}$ within targeted and general trials (columns 6-8 and 9-11) are not equal to the corresponding number of trials under targeted and general in the left side of the table (columns 4 and 5).

Targeted trials with an objective $(O)$ defined by PROGRESS-Plus factors were further scrutinized for different PROGRESS-Plus factors in their baseline characteristics $(B)$ or analysis $(A)$ - the inclusion of a PROGRESS-Plus factor different from that targeted by the intervention objective $(\mathrm{O})$ would warrant the trial to be counted under headings $B$ and $A$ in their respective PROGRESS-Plus categories.

differential effects among disadvantaged subgroups when intervention outcomes were further analysed. Notably, six trials found differences in outcomes based on the sex of participants, five trials found differences based on age and four trials found differences based on race/ethnicity group.

Table 3 examines the extent of equity-relevance in diabetes QI trials over time. Prior to 2007, there were

Table 3 Frequency of PROGRESS-Plus factors stratified by year of study conduct

\begin{tabular}{|c|c|c|c|c|c|c|c|c|}
\hline \multirow{3}{*}{$\begin{array}{l}\text { PROGRESS-Plus } \\
\text { factors } \\
\text { Year of study } \\
\text { conduct }\end{array}$} & \multirow{2}{*}{\multicolumn{2}{|c|}{$\begin{array}{l}\text { Non-equity studies, } \\
\mathrm{n}=177\end{array}$}} & \multicolumn{6}{|c|}{ Equity-relevant studies, n=95 } \\
\hline & & & \multicolumn{2}{|l|}{ All, $n=95$} & \multicolumn{2}{|c|}{ Targeted, $\mathrm{n}=64$} & \multicolumn{2}{|c|}{ General, $n=31$} \\
\hline & $\begin{array}{l}\text { Pre-2007, } \\
\mathrm{n}=85\end{array}$ & $\begin{array}{l}2007-2014 \\
n=92\end{array}$ & $\begin{array}{l}\text { Pre-2007, } \\
\mathrm{n}=41\end{array}$ & $\begin{array}{l}2007-2014 \\
n=54\end{array}$ & $\begin{array}{l}\text { Pre-2007, } \\
\mathrm{n}=23\end{array}$ & $\begin{array}{l}2007-2014, \\
n=41\end{array}$ & $\begin{array}{l}\text { Pre-2007, } \\
\mathrm{n}=18\end{array}$ & $\begin{array}{l}2007-2014, \\
n=13\end{array}$ \\
\hline Place of residence & $2(2.4)$ & $2(2.2)$ & $10(24.4)$ & $9(16.7)$ & $10(43.5)$ & $9(22)$ & $0(0)$ & $0(0)$ \\
\hline $\begin{array}{l}\text { Race/ethnicity/ } \\
\text { culture/language }\end{array}$ & 28 (32.9) & $34(37)$ & $31(75.6)$ & $44(81.5)$ & $20(87)$ & $37(90.2)$ & $11(61.1)$ & $7(53.8)$ \\
\hline Occupation & $5(5.9)$ & $12(13)$ & $6(14.6)$ & 18 (33.3) & $5(21.7)$ & $13(31.7)$ & $1(5.6)$ & $5(38.5)$ \\
\hline Gender/sex & $72(84.7)$ & $88(95.7)$ & $39(95.1)$ & $52(96.3)$ & $21(91.3)$ & $39(95.1)$ & $18(100)$ & $13(100)$ \\
\hline Religion & $0(0)$ & $0(0)$ & $1(2.4)$ & $0(0)$ & $1(4.3)$ & $0(0)$ & $0(0)$ & $0(0)$ \\
\hline Education & $21(24.7)$ & $36(39.1)$ & $18(43.9)$ & $39(72.2)$ & $11(47.8)$ & $31(75.6)$ & $7(38.9)$ & $8(61.5)$ \\
\hline $\begin{array}{l}\text { Socioeconomic } \\
\text { status }\end{array}$ & $13(15.3)$ & $14(15.2)$ & $18(43.9)$ & $33(61.1)$ & $16(69.6)$ & $29(70.7)$ & $2(11.1)$ & $4(30.8)$ \\
\hline Social capital & $10(11.8)$ & $20(21.7)$ & $11(26.8)$ & $22(40.7)$ & $9(39.1)$ & $21(51.2)$ & $2(11.1)$ & $1(7.7)$ \\
\hline Plus 1. Age & 78 (91.8) & $89(96.7)$ & $40(97.6)$ & $52(96.3)$ & $22(95.7)$ & $39(95.1)$ & $18(100)$ & $13(100)$ \\
\hline Plus 1. Disability & $24(28.2)$ & 29 (31.5) & $6(14.6)$ & $26(48.1)$ & $3(13)$ & $20(48.8)$ & $3(16.7)$ & $6(46.2)$ \\
\hline
\end{tabular}

Year of publication where the last year of study conduct was not reported. All values are expressed as $\mathrm{n}(\%)$. 
41 equity-relevant trials out of 126 diabetes QI studies. In the period from 2007 to 2014, there were 54 equity-relevant trials out of 146 diabetes QI studies. Targeted trials were responsible for $56.1 \%$ of equity-relevant trials prior to 2007. This number increased to $75.9 \%$ of equity-relevant trials in the period from 2007 to 2014. The most notable increases in the absolute number of targeted studies occurred with occupation, education and disability.

\section{CONCLUSIONS}

Among 278 diabetes QI trials, only $34.9 \%$ provided equity-relevant findings. These studies provide insight into the effectiveness of various diabetes management strategies among racial and ethnic minorities, among patients with low income or low socioeconomic status, as well as in remote medically underserved populations. A few trials looked at age, gender/sex, education status and disability as potential factors that contribute to disparities in diabetes care. However, we know little about the effects of other factors that may play a role in determining how patients experience and respond to health interventions.

Trials designed for the general population which then conducted stratified analyses point to the importance of considering PROGRESS-Plus factors when designing and examining interventions. In fact, QI strategies designed for the general population may not be accessible to disadvantaged groups or may not have the same efficacy in disadvantaged populations and may inadvertently lead to an increase in diabetes-related health disparities. For example, a general trial looking at telehome monitoring systems as an affordable and efficient means to monitor patients with diabetes showed greater efficacy in patients who were male, elderly and/or more educated. ${ }^{15}$ Another study examining the efficacy of telephone-based weight loss programmes to improve glycaemic control found the intervention outcomes differed between Caucasians and non-Caucasians. ${ }^{18}$ Alternatively, adding care guides to the primary care team was more likely to have benefits for patients on Medicaid rather than patients with other forms of health insurance in the USA. ${ }^{19}$ These findings emphasise the need for better data collection, reporting and analysis on the social determinants of health that may influence the health outcomes of patients with diabetes.

While the majority of diabetes QI trials did not have an equity focus, the vast majority collected some form of equity-relevant data to assess balance between study arms in RCTs. This collection of stratified data presents a missed opportunity for researchers to further explore equity-relevant analyses.

In this systematic review, only 22 trials $(8.1 \%)$ directed interventions toward a population that was impacted by two or more risk factors for health disparities. The limited foci of diabetes QI trials stand in contrast to the harsh reality of many patients, for whom multiple social and economic determinants of health intersect in complex ways. The risk for health disparities often increases in populations where multiple PROGRESS-Plus factors are concerned. ${ }^{9}$ For example, there is a paucity of research targeting elderly racial and ethnic minorities despite this being one of the fastest growing demographic for diabetes diagnoses and diabetes-related complications. ${ }^{3}$ Given that these populations tend to bear a disproportionate burden of disease, ${ }^{23}$ it is even more important that interventions and analyses relevant to these patients be represented in the current body of research.

Interventions tailored toward socially disadvantaged populations show promising results in reducing health disparities in diabetes care. In a review of 17 QI trials, Glazier et $a l^{2}$ found that interventions worked best when they were adapted to the local community to fit local circumstances. Similarly, in a study of 42 QI trials, Peek $e t a l^{3}$ found evidence to suggest that culturally tailored programming and community-based partnerships led to improvements in health outcomes for racial/ ethnic minorities and successfully contributed to reductions in health disparities in the population. Fisher et $a t^{20}$ showed narrowing of racial disparities in healthcare with culturally specific programming and healthcare messaging. Culturally sensitive strategies can help strengthen connections between patients and healthcare organisations, thereby facilitating a more effective exchange of health information and improved adherence to treatment plans for ethnic and racial minorities.

In 2013, Clarke et al reported that interventions to improve care in ethnic minorities predominantly focused on patient-level strategies, placing the burden of change on patients without addressing equally relevant factors at the level of health providers, healthcare organisations and health systems. ${ }^{11}$ Here, we show that there is increasing data from trials testing health system interventions, such as case management and team changes, to consider when developing QI interventions to either address or prevent worsening health inequities across several PROGRESS-Plus characteristics.

This study has several limitations. First, we included only English language publications as translation of non-English studies was not feasible. Second, due the large number of included studies we focused our review of equity factors in the primary publication of trials. It is possible that authors did additional equity-relevant analyses in secondary publications, which were not captured in this review. This may result in an underestimate of the number of general studies that analysed effects in disadvantaged groups. However, by focusing on primary publication, we have identified studies in which equity concerns were prioritised by authors, either through targeted interventions or subsequent analyses, to warrant discussion in a primary paper. Finally, our ability to capture the full breadth of issues that may disadvantage patients was restricted by limitations in reporting these variables within each study and by the lack of a standardised terminology in the literature.

As our objective was to assess the extent to which researchers considered equity-relevant factors, we did not analyse the effect of equity-relevant factors on study 
outcomes. The effectiveness of interventions often varies based on the participant population and the existing health system. Based on the limited primary data that we have from targeted and general trials, it is difficult to make definitive conclusions about which interventions or QI strategies are effective or ineffective in reducing health disparities and/or improving health outcomes for disadvantaged groups without oversimplifying the issue and potentially misleading future enquiry. What ultimately works in one healthcare setting may not work in another. However, this represents an important area for future research.

In conclusion, the findings in this secondary study of a systematic review of diabetes QI trials indicate substantial room for improvement in the proportion of studies that address equity and the range of equity factors that can be reported and analysed.

\section{Author affiliations}

${ }^{1}$ Faculty of Health Sciences, School of Medicine, Queen's University, Kingston, Ontario, Canada

${ }^{2}$ Clinical Epidemiology Program, Ottawa Hospital Research Institute, Ottawa, Ontario, Canada

${ }^{3}$ Faculty of Medicine, School of Epidemiology, Public Health and Preventive Medicine, University of Ottawa, Ottawa, Ontario, Canada

${ }^{4}$ Faculty of Medicine, University of Toronto, Toronto, Ontario, Canada

${ }^{5}$ Bruyère Research Institute, Ottawa, Ontario, Canada

${ }^{6}$ Department of Medicine, Faculty of Medicine, University of Ottawa, Ottawa, Ontario, Canada

${ }^{7}$ Family Practice Health Centre, Women's College Research Institute, Toronto, Ontario, Canada

${ }^{8}$ Institute for Health Systems Solutions and Virtual Care, Women's College Hospital, Toronto, Ontario, Canada

${ }^{9}$ Department of Family and Community Medicine, Dalla Lana School of Public Health, Institute of Health Policy Management and Evaluation, University of Toronto, Toronto, Ontario, Canada

Acknowledgements The authors wish to thank Jennifer Petkovic at the Bruyère Research Institute for her valuable feedback during the design of this study.

Contributors NMI and KJD conceived the study. All authors participated in the design of the study. KJD managed and coordinated the source data. JBL and MDE coded the source data with input from NMI and KJD to resolve discrepancies between coders. All authors contributed to the interpretation of the findings. Writing of the paper was led by JBL, KJD and NMI with MDE, VW and JMG commenting on drafts. All authors approved the final manuscript.

Funding This study is supported by a grant from the Canadian Institutes of Health Research (CIHR, FRN-123345). KJD holds a Frederick Banting and Charles Best Canada Graduate Scholarship. VW holds an Ontario Early Researcher Award. NMI is funded by a New Investigator Award from the Canadian Institutes of Health Research. JMG holds a Canada Research Chair in Health Knowledge Transfer and Uptake and is funded by a Foundation Grant from the Canadian Institutes of Health Research.

Disclaimer CIHR had no role in the study design, plans for data collection and analysis, decision to publish or preparation of this research.

Competing interests None declared.

Patient consent Not required.

Provenance and peer review Not commissioned; externally peer reviewed.

Data sharing statement The dataset generated and analysed during the current study are available from the corresponding author on request.

Open Access This is an Open Access article distributed in accordance with the Creative Commons Attribution Non Commercial (CC BY-NC 4.0) license, which permits others to distribute, remix, adapt, build upon this work non-commercially, and license their derivative works on different terms, provided the original work is properly cited and the use is non-commercial. See: http://creativecommons.org/ licenses/by-nc/4.0/

(C) Article author(s) (or their employer(s) unless otherwise stated in the text of the article) 2018. All rights reserved. No commercial use is permitted unless otherwise expressly granted.

\section{REFERENCES}

1. World Health Organization. Global report on diabetes. France: World Health Organization, 2016.

2. Glazier RH, Bajcar J, Kennie NR, et al. A systematic review of interventions to improve diabetes care in socially disadvantaged populations. Diabetes Care 2006;29:1675-88.

3. Peek ME, Cargill A, Huang ES. Diabetes health disparities: a systematic review of health care interventions. Med Care Res Rev 2007;64:101S.

4. Cheng AY. Canadian Diabetes Association Clinical Practice Guidelines Expert Committee. Canadian Diabetes Association 2013 clinical practice guidelines for the prevention and management of diabetes in Canada. Introduction. Can J Diabetes 2013;37(suppl 1):S1-212.

5. American Diabetes Association. Foundations of care and comprehensive medical evaluation. Diabetes Care 2016;39(Suppl 1):S23-35.

6. Borkhoff CM, Wieland ML, Myasoedova E, et al. Reaching those most in need: a scoping review of interventions to improve health care quality for disadvantaged populations with osteoarthritis. Arthritis Care Res 2011;63:39-52.

7. Chin MH, Parrillo R. Advancing equity in healthcare: lessons from Massachusetts and beyond. BMJ 2015;350:h1617.

8. Chin MH, Goddu AP, Ferguson MJ, et al. Expanding and sustaining integrated health care-community efforts to reduce diabetes disparities. Health Promot Pract 2014;15:29S-39.

9. O'Neill J, Tabish $\mathrm{H}$, Welch $\mathrm{V}$, et al. Applying an equity lens to interventions: using PROGRESS ensures consideration of socially stratifying factors to illuminate inequities in health. $J$ Clin Epidemiol 2014;67:56-64

10. Tricco AC, Ivers NM, Grimshaw JM, et al. Effectiveness of quality improvement strategies on the management of diabetes: a systematic review and meta-analysis. Lancet 2012;379:2252-61.

11. Clarke AR, Goddu AP, Nocon RS, et al. Thirty years of disparities intervention research: what are we doing to close racial and ethnic gaps in health care? Med Care 2013;51-1020-6.

12. Lorenc $\mathrm{T}$, Petticrew $\mathrm{M}$, Welch $\mathrm{V}$, et al. What types of interventions generate inequalities? Evidence from systematic reviews. $J$ Epidemiol Community Health 2013;67:190-3.

13. Ivers N, Tricco AC, Trikalinos TA, et al. Seeing the forests and the trees-innovative approaches to exploring heterogeneity in systematic reviews of complex interventions to enhance health system decisionmaking: a protocol. Syst Rev 2014;3:88.

14. Gary TL, Bone LR, Hill MN, et al. Randomized controlled trial of the effects of nurse case manager and community health worker interventions on risk factors for diabetes-related complications in urban African Americans. Prev Med 2003;37:23-32.

15. Bujnowska-Fedak MM, Puchała E, Steciwko A. The impact of telehome care on health status and quality of life among patients with diabetes in a primary care setting in Poland. Telemed J E Health 2011;17:153-63.

16. DePue JD, Dunsiger S, Seiden AD, et al. Nurse-community health worker team improves diabetes care in American Samoa: results of a randomized controlled trial. Diabetes Care 2013;36:1947-53.

17. Shea S, Weinstock RS, Teresi JA, et al. A randomized trial comparing telemedicine case management with usual care in older, ethnically diverse, medically underserved patients with diabetes mellitus: 5 year results of the IDEATel study. J Am Med Inform Assoc 2009;16:446-56.

18. Eakin EG, Reeves MM, Winkler E, et al. Six-month outcomes from living well with diabetes: A randomized trial of a telephone-delivered weight loss and physical activity intervention to improve glycemic control. Ann Behav Med 2013;46:193-203.

19. Adair R, Wholey DR, Christianson J, et al. Improving chronic disease care by adding laypersons to the primary care team: a parallel randomized trial. Ann Intern Med 2013;159:176-84.

20. Fisher TL, Burnet DL, Huang ES, et al. Cultural leverage: interventions using culture to narrow racial disparities in health care. Med Care Res Rev 2007;64:243S-82. 